Het er osynapt i c expressi on of depol ar i zat i on- i nduced suppr essi on of i nhi bi ti on (DSI) i $n$ rat hi ppocampal cul tures

\begin{tabular}{|l|l|}
\hline 著者 & $\begin{array}{l}\text { Ohno- Shosaku Takako, Sawada Sat suki, Kano } \\
\text { Nasanobu }\end{array}$ \\
\hline $\begin{array}{l}\text { j our nal or } \\
\text { publ i cat i on ti t l e }\end{array}$ & Neur osci ence Resear ch \\
\hline vol une & 36 \\
\hline number & 1 \\
\hline page r ange & $67-71$ \\
\hline year & $2000-01-01$ \\
\hline URL & ht t p: //hdl . handl e. net /2297/1657 \\
\hline
\end{tabular}




\section{Heterosynaptic expression of depolarization-induced suppression of inhibition (DSI) in rat hippocampal cultures}

Takako Ohno-Shosaku ${ }^{1}$, Satsuki Sawada ${ }^{1}$ and Masanobu Kano ${ }^{1,2}$

${ }^{1}$ Department of Physiology, Kanazawa University School of Medicine, Kanazawa 920-8640, Japan

${ }^{2}$ CREST, Japan Science and Technology Corporation

\section{Correspondence :}

T. Ohno-Shosaku

Department of Physiology, Kanazawa University School of Medicine, Takara-machi, Kanazawa 920-8640, Japan

TEL : 81-76-265-2171

FAX : 81-76-234-4224

E-mail :shosaku@med.kanazawa-u.ac.jp

Key Words:

Inhibitory transmission - Hippocampal neuron - DSI - Heterosynaptic modulation Depolarization - Transient suppression 


\section{ABSTRACT}

Depolarization-induced suppression of inhibition (DSI) is a transient suppression of the inhibitory synaptic transmission observed in the hippocampus and the cerebellum upon postsynaptic depolarization. Using rat hippocampal cultures, we examined whether DSI is confined to the inhibitory synapses on the depolarized neuron or DSI can spread to those on neighboring non-depolarized neurons. Whole-cell recordings were performed in 108 neuronal pairs with the following synaptic responses. Stimulation of one neuron evoked the inhibitory autaptic currents (IACs) recurrently in that neuron and also elicited the inhibitory postsynaptic currents (IPSCs) orthodromically in the other neuron. In 38 of 108 pairs, the postsynaptic depolarization caused transient suppression of IPSCs (homosynaptic DSI). In 11 of the 38 pairs exhibiting the homosynaptic DSI, the depolarization also induced suppression of IACs (heterosynaptic DSI). The heterosynaptic DSI, like the homosynaptic DSI, depended on depolarizing pulse duration and was blocked by a phorbol ester. These results suggest that DSI can spread to the synapses on a neighboring non-depolarized neuron in rat hippocampal cultures.

\section{INTRODUCTION}

Depolarization of a cerebellar Purkinje cell (Llano et al. 1991; Vincent et al. 1992) or a hippocampal CA1 pyramidal cell (Pitler and Alger, 1992) induces transient suppression of inhibitory synaptic inputs to the depolarized cell. This phenomenon, known as depolarization-induced suppression of inhibition (DSI), is reproducible in a single pair of neurons in rat hippocampal cultures (Ohno-Shosaku et al. 1998). As for DSI mechanisms, considerable evidence suggests that depolarization causes intracellular $\mathrm{Ca} 2+$ elevation by activating $\mathrm{Ca} 2+$ channels, which in turn suppresses the inhibitory transmission presynaptically via retrograde signals (Pitler and Alger, 1994; Alger and Pitler, 1995; Lenz et al. 1998). Recent reports have proposed that glutamate or a glutamate-like substance might be the retrograde messenger that mediates DSI in the cerebellum (Glitsch et al. 1996) and the hippocampus (Morishita et al. 1998). In the hippocampus, DSI or DSI-like processes can be activated by endogenous burst of firing of CA1 pyramidal cells (Le Beau and Alger 1998). This suggests that DSI could occur 
under certain physiological conditions and thus plays an important role in information processing in the hippocampus.

Several previous studies demonstrated the spread of activity-dependent synaptic modifications to neighboring synapses that have not been activated during its induction. In the hippocampus, long-term depression (LTD) or long-term potentiation (LTP) is reported to spread to neighboring excitatory synapses (Schuman and Madison, 1994; Scanziani et al. 1996; Fitzsimonds et al. 1997). The spread of DSI in the hippocampus, however, has never been reported.

In the present study, we examined whether DSI can spread to neighboring inhibitory synapses on a non-depolarized neuron, using rat hippocampal cultures. We found, for the first time, that a spread of DSI can occur in hippocampal neurons. Possible mechanisms for the spread will be discussed.

\section{MATERIALS AND METHODS}

Hippocampal neurons were prepared from newborn rats and cultured for 7-14 days as described previously (Ohno-Shosaku et al. 1998). All experiments were performed at room temperature. The external solution contained (in $\mathrm{mM}$ ) $140 \mathrm{NaCl}, 2.5$ $\mathrm{KCl}, 1 \mathrm{MgCl} 2,2 \mathrm{CaCl} 2,10$ HEPES, 10 glucose and 1 kynurenic acid (pH 7.3 adjusted with $\mathrm{NaOH}$ ). The internal solution contained $120 \mathrm{~K}$-gluconate, $15 \mathrm{KCl}, 6 \mathrm{MgCl} 2,5$ EGTA, 10 HEPES, $20 \mathrm{KOH}$ and $5 \mathrm{Na} 2 \mathrm{ATP}$ (pH 7.3 adjusted with $\mathrm{KOH}$ ). The electrode resistance ranged from 3 to $5 \mathrm{M} \Omega$ when filled with the solution.

A pair of neurons were whole-cell clamped with two different patch pipettes, and membrane potentials of both cells were held at $-80 \mathrm{mV}$. The presynaptic neuron was stimulated by applying positive voltage-pulses $(80 \mathrm{mV}, 2 \mathrm{msec})$ at $0.2 \mathrm{~Hz}$, and synaptic currents were measured with a patch-clamp amplifier (EPC-7/List or EPC-9/HEKA, Germany). Excitatory and inhibitory currents were identified from their kinetic properties and reversal potentials.

For the induction of DSI, the postsynaptic neuron was depolarized to $0 \mathrm{mV}$ for $5 \mathrm{sec}$, unless otherwise noted. To estimate the suppression of synaptic currents, the mean amplitude of three consecutive synaptic currents acquired between 6 and $16 \mathrm{sec}$ after the end of depolarization was expressed as the percentage of the mean amplitude of five synaptic currents before the depolarization. A "DSI-positive" pair indicates a pair 
exhibiting more than $15 \%$ decrease in amplitudes of synaptic currents after the depolarization for $5 \mathrm{sec}$. Averaged data from different experiments are presented as mean \pm SEM.

Kynurenic acid and PDAc (phorbol 12, 13-diacetate) were purchased from Wako (Osaka, Japan), and bicuculline from Sigma (St. Louis, MO).

\section{RESULTS}

In this study, we used 108 pairs of neurons with the connectivity illustrated in Fig. 1. Each pair was composed of an inhibitory presynaptic neuron and the synaptically connected postsynaptic neuron. The presynaptic neuron was stimulated, and the evoked inhibitory autaptic currents (IACs) and the inhibitory postsynaptic currents (IPSCs) were recorded from the presynaptic and the postsynaptic neurons, respectively. Bath application of TTX, Cd2+, or bicuculline eliminated these currents (Fig. 1), thus confirming that they were GABAA receptor-mediated inhibitory synaptic currents.

\section{Homosynaptic and heterosynaptic DSIs}

When the postsynaptic neuron was depolarized to $0 \mathrm{mV}$ for $5 \mathrm{sec}$, the IPSCs recorded from the depolarized neuron were transiently suppressed (Figs. 2 and 3A) in 38 of 108 pairs tested. We call this suppression of IPSCs "homosynaptic DSI." In 11 of the 38 pairs exhibiting the homosynaptic DSI, depolarization also induced transient suppression of IACs, which we call "heterosynaptic DSI." Figure 2A shows an example of the results obtained from such a pair. The time course of the heterosynaptic DSI was similar to that of the homosynaptic DSI (Fig. 2B). In 10 out of 11 pairs, the magnitude of heterosynaptic DSI was smaller than that of the homosynaptic DSI (Fig. 2C). On the average, the amplitude of IPSCs decreased by $74.1 \pm 8.8 \%$ after depolarization, and that of IACs by $37.9 \pm 5.7 \%$. In the remaining 27 of the 38 pairs, IACs were not affected by depolarization (Fig. 3A). In this group, the decrease in IPSC amplitudes ranged from $15 \%$ to $99 \%$, and averaged $59.5 \pm 4.9 \%$. These values were comparable with those obtained in the pairs showing the heterosynaptic DSI (Fig. 2C). Thus, the lack of heterosynaptic DSI in these pairs seems unlikely to result from insufficient production of retrograde signals at the induction site.

In 70 of 108 pairs, the homosynaptic DSI was not induced, which is in good 
agreement with our previous observation showing that $62 \%$ of pairs were DSI-negative (Ohno-Shosaku et al. 1998). In these pairs, IACs were never suppressed after depolarization (Fig. 3B).

\section{Properties of heterosynaptic DSI}

Properties of the heterosynaptic DSI were further examined, using the pairs exhibiting both the homosynaptic and the heterosynaptic DSIs. First, we examined the dependence of DSI on the duration of depolarization was examined (Fig. 4). When the duration of postsynaptic depolarization was decreased from $5 \mathrm{sec}$ to $1-4 \mathrm{sec}$, the magnitude of the heterosynaptic DSI was decreased accordingly (Fig. 4, closed column). This duration-dependence of the heterosynaptic DSI was similar to that of the homosynaptic DSI (Fig. 4, open column). Next, effects of PDAc-treatment on DSI were examined (Fig. 5). Application of PDAc to the bath solution strongly suppressed the heterosynaptic DSI as well as the homosynaptic DSI. The effects of PDAc were irreversible and both DSIs were not recovered after washout of PDAc. These results suggest that the heterosynaptic and the homosynaptic DSIs may share the same mechanisms.

\section{DISCUSSION}

The present study demonstrated that depolarization of one neuron can induce suppression of the inhibitory input to another, non-depolarized neuron. This phenomenon, termed here as "heterosynaptic DSI", is a new finding for hippocampal neurons, although a similar spread of DSI, termed "cross-inhibition," has been reported in the cerebellum (Vincent and Marty, 1993). The heterosynaptic DSI depended on the duration of postsynaptic depolarization and was blocked by a phorbol ester. These properties are similar to those of the homosynaptic DSI (Ohno-Shosaku et al. 1998), suggesting that both phenomena would involve the same mechanisms.

Possible mechanisms, by which DSI spreads to the autapses of the presynaptic neuron, are as follows. (1) A retrograde messenger (m) released from the depolarized postsynaptic neuron diffuses to the autapses (Fig. 6A). (2) The retrograde messenger acts on the presynaptic terminals located on the depolarized neuron and a suppressing signal (s) is then propagated to the autaptic terminals through the axon (Fig. 6B). (3) The 
retrograde messenger causes a conduction block by acting on the axons, whose branches project to both the presynaptic and the postsynaptic neurons (Fig. 6C). (4) The first retrograde messenger $(\mathrm{m} 1)$ released from the depolarized postsynaptic neuron activates a glial cell, which in turn triggers DSI by releasing the second substance (m2) (Fig. 6D). (5) IACs are generated by activation of GABAA receptors located, not on dendrites or cell bodies, but on presynaptic terminals (Fig. 6E). If this type of transmission, called "cis-mission" (Vautrin et al. 1994), contributes to the IACs recorded here, the decrease in GABA release in presynaptic terminals will also lead to the decrease in IAC amplitudes. (6) IPSCs and IACs are generated by GABA release from the same presynaptic bouton, referred to as multiple-synapse bouton (MSB) (Fig. 6F). If an intracellular signal is generated at the presynaptic site facing the depolarized neuron, it will spread easily within MSB, and thereby cause suppression of transmitter release from the same MSB to the non-depolarized neuron. A similar model has been proposed to account for the interneuronal spread of LTP (Harris, 1995). At present, we have no experimental evidence that positively supports any of these possibilities.

Triple whole-cell recordings from cultured hippocampal neurons (Fitzsimonds et al. 1997) demonstrated that LTD at glutamatergic synapses spreads to the synapses on the presynaptic neuron (back propagation) and to the output synapses of the common presynaptic neuron (lateral propagation), but not to the output of the postsynaptic neuron (forward propagation). This suggests a selective spread of synaptic modification in the neural network. In the study presented here, we demonstrated that postsynaptic depolarization can induce suppression of the autaptic currents in the presynaptic neuron, which corresponds to back/lateral propagation of DSI. It would be interesting to test whether DSI also exhibits forward propagation. Whether the spread of DSI is selective or not would be important for understanding the mechanisms and functional roles of DSI.

\section{ACKNOWLEDGEMENTS}

This work was supported by Grants-in Aid for Scientific Research (09680813, 10480230, 11670036) from the Ministry of Education, Science, Sports and Culture of Japan, by Human Frontier Science Program, and by Special Coordination Funds for Promoting Science and Technology from STA. The authors are grateful to Mrs. Y. Aoyagi for her editorial assistance. 


\section{REFERENCES}

Alger, B. E. and Pitler, T. A. (1995) Retrograde signaling at GABAA-receptor synapses in the mammalian CNS. Trends in Neurosci., 18: 333-340.

Fitzsimonds, R. M., Song, H.-j. and Poo, M. m. (1997) Propagation of activity-dependent synaptic depression in simple neural networks. Nature, 388: 439-448.

Glitsch, M., Llano, I. and Marty, A. (1996) Glutamate as a candidate retrograde messenger at interneurone-Purkinje cell synapses of rat cerebellum. J. Physiol., 497: 531-537.

Harris, K. M. (1995) How multiple-synapse boutons could preserve input specificity during an interneuronal spread of LTP. Trends in Neurosci., 18: 365-369.

Le Beau, F. E. N. and Alger, B. E. (1998) Transient suppression of GABAA-receptor-mediated IPSPs after epileptiform burst discharges in CA1 pyramidal cells. J. Neurophysiol., 79: 659-669.

Lenz, R. A., Wagner, J. J., Alger, B. E. (1998) N- and L-type calcium channel involvement in depolarization-induced suppression of inhibition in rat hippocampal CA1 cells. J. Physiol., 512: 61-73.

Llano, I., Leresche, N. and Marty, A. (1991) Calcium entry increases the sensitivity of cerebellar Purkinje cells to applied GABA and decreases inhibitory synaptic currents. Neuron, 6: 565-574.

Morishita, W., Kirov, S. A. and Alger, B. E. (1998) Evidence for metabotropic glutamate receptor activation in the induction of depolarization-induced suppression of inhibition in hippocampal CA1. J. Neurosci., 18: 4870-4882.

Ohno-Shosaku, T., Sawada, S. and Yamamoto, C. (1998) Properties of depolarization-induced suppression of inhibitory transmission in cultured rat hippocampal neurons. Pfluegers Arch., 435: 273-279.

Pitler, T. A. and Alger, B. E. (1992) Postsynaptic spike firing reduces synaptic GABAA responses in hippocampal pyramidal cells. J. Neurosci., 12: 4122-4132.

Pitler, T. A. and Alger, B. E. (1994) Depolarization-induced suppression of GABAergic inhibition in rat hippocampal pyramidal cells: $G$ protein involvement in a presynaptic mechanism. Neuron, 13: 1447-1455.

Scanziani, M., Malenka, R. C. and Nicoll, R. A. (1996) Role of intercellular interactions in heterosynaptic long-term depression. Nature, 380: 446-450.

Schuman, E. M. and Madison, D. V. (1994) Locally distributed synaptic potentiation in 
the hippocampus. Science, 263: 532-536.

Vautrin, J., Schaffner, A. E. and Barker, J. L. (1994) Fast presynaptic GABAA receptor-mediated $\mathrm{Cl}$ - conductance in cultured rat hippocampal neurones. J. Physiol., 479: 53-63.

Vincent, P., Armstrong, C. M. and Marty, A. (1992) Inhibitory synaptic currents in rat cerebellar Purkinje cells: Modulation by postsynaptic depolarization. J. Physiol., 456: 453-471.

Vincent, P. and Marty, A. (1993) Neighboring cerebellar Purkinje cells communicate via retrograde inhibition of common presynaptic interneurons. Neuron, 11: 885-893. 


\section{FIGURE LEGENDS}

Fig. 1. An example of simultaneous recordings of IACs and IPSCs. A: An inhibitory presynaptic neuron was stimulated by a voltage pulse (V1), and the evoked IAC (I1) and IPSC (I2) were recorded from the presynaptic neuron (1) and a postsynaptic neuron (2), respectively. B-D: Both IAC and IPSC were abolished by extracellularly applied $0.2 \mu \mathrm{M}$ TTX (B), $0.1 \mathrm{mM} \mathrm{Cd}^{2+}(\mathrm{C})$ or $10 \mu \mathrm{M}$ bicuculline (D).

Fig. 2. Effects of postsynaptic depolarization on IPSCs and IACs in the pairs exhibiting both homosynaptic and heterosynaptic DSIs. A: A typical example of depolarization-induced suppression of IPSCs (left) and of IACs (right), both of which were measured simultaneously. The postsynaptic neuron was depolarized for $5 \mathrm{sec}$ at the time indicated by the arrows. The traces in the upper panel are examples of IPSCs and IACs acquired at the time indicated by a-c in the graphs. B: Averaged time course of depolarization-induced suppression of IPSCs (open circles) and that of IACs (closed circles) obtained from 11 pairs. C: The percentage decreases in amplitudes of IPSCs and IACs. Each line connects points obtained from the same pair.

Fig. 3. Effects of postsynaptic depolarization on IPSCs and IACs in the pairs exhibiting no heterosynaptic DSI. A: Data from 27 pairs exhibiting homosynaptic DSI but not heterosynaptic DSI. B: Data from 15 pairs randomly selected from 70 DSI-negative pairs. Graphs are illustrated as in Fig. 2B.

Fig. 4. The relationship between the duration of depolarization and the magnitude of homosynaptic (open column, $n=4-6$ ) or heterosynaptic (closed column, $n=4-6$ ) DSI. The magnitude of suppression was normalized to the value obtained with 5-sec-depolarization.

Fig. 5. Effects of PDAc on heterosynaptic DSI (IAC) and homosynaptic DSI (IPSC). A: The traces are examples of IACs and IPSCs acquired before and $6 \mathrm{sec}$ after depolarization in the presence or absence of $10 \mu \mathrm{M}$ PDAc. B: The percentage decreases in amplitude of IACs and IPSCs before and after addition of PDAc were obtained from three pairs. 
Fig. 6. Possible mechanisms for heterosynaptic DSI. A: Spread of a retrograde messenger $(\mathrm{m})$ from the depolarized neuron to the autapses. B: Propagation of a suppressing signal (s) through the axon. C: Axonal conduction block mediated by a retrograde signal (m). D: Glia-neuron interaction. E: Contribution of cis-mission to IACs. F: Involvement of multiple-synapse bouton. 


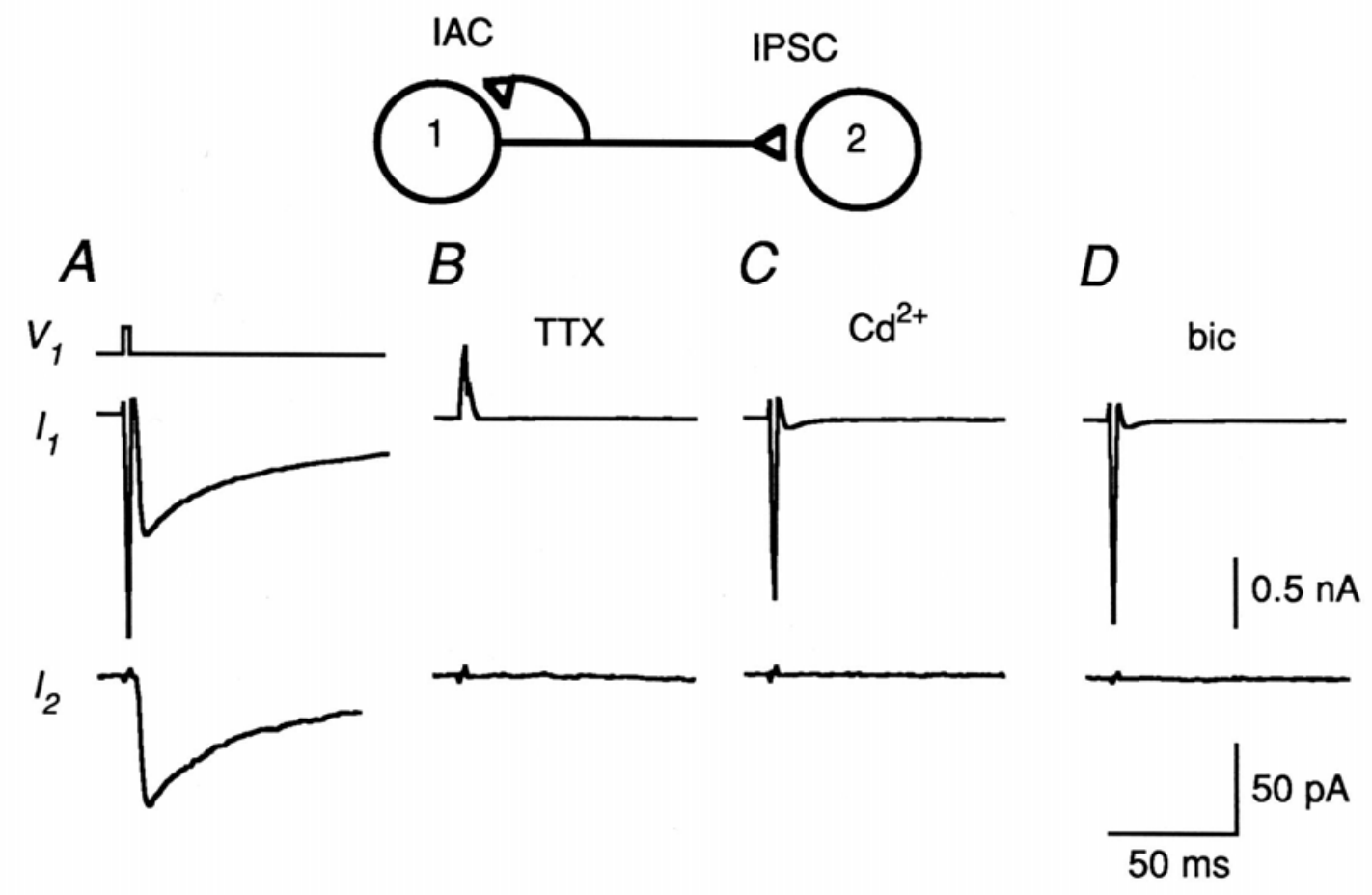

Figure 1 

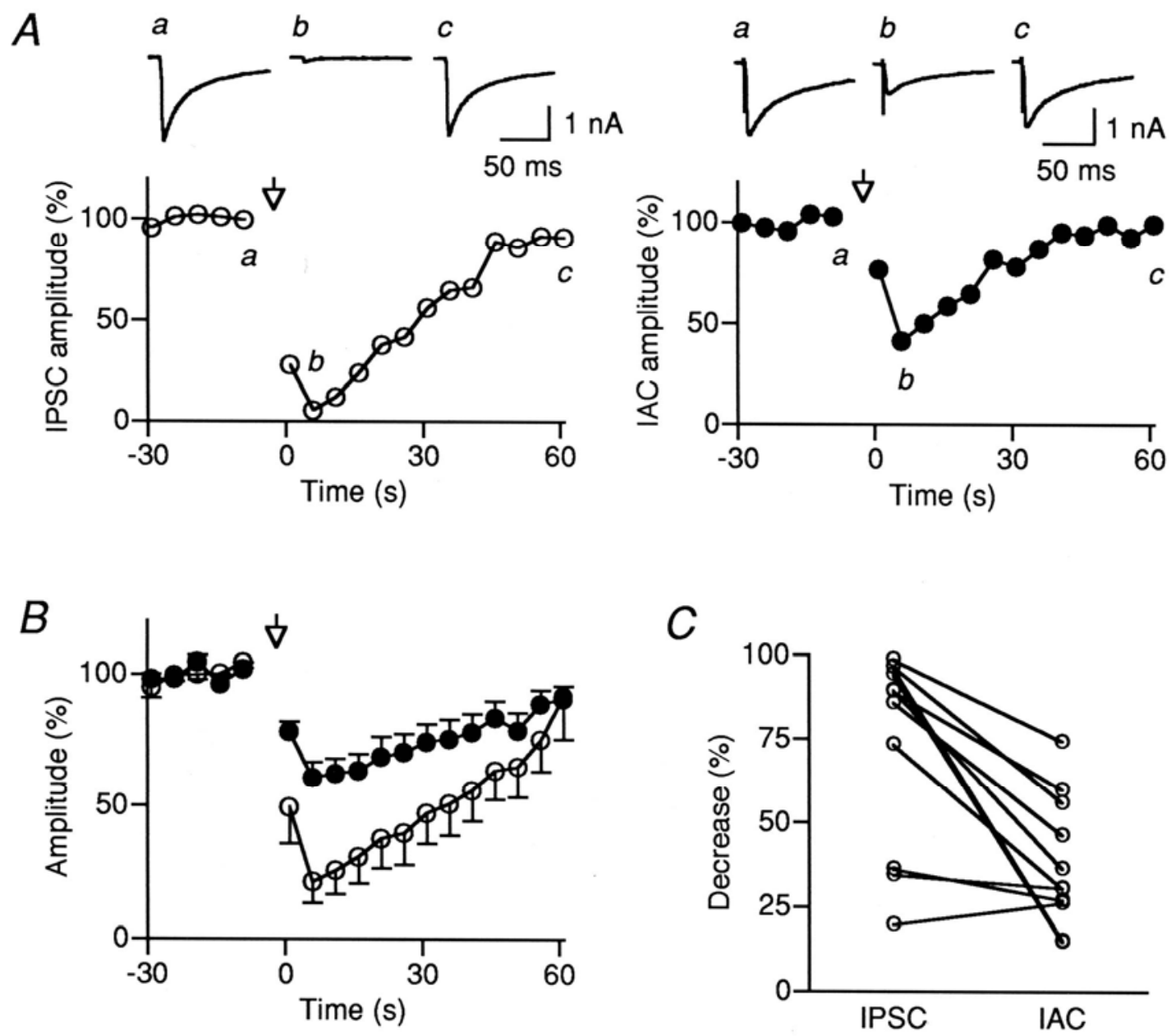

Figure 2 

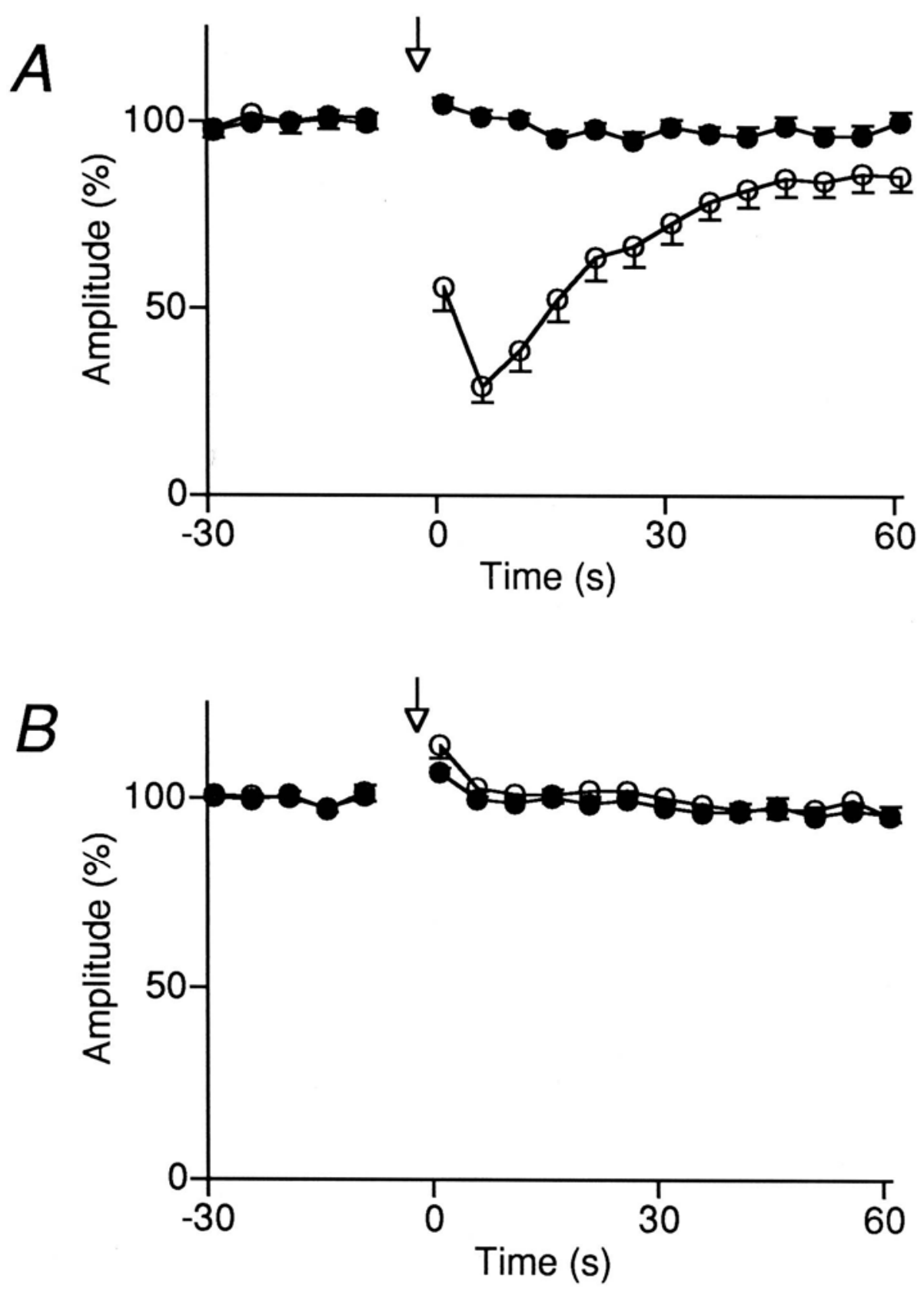

Figure 3 


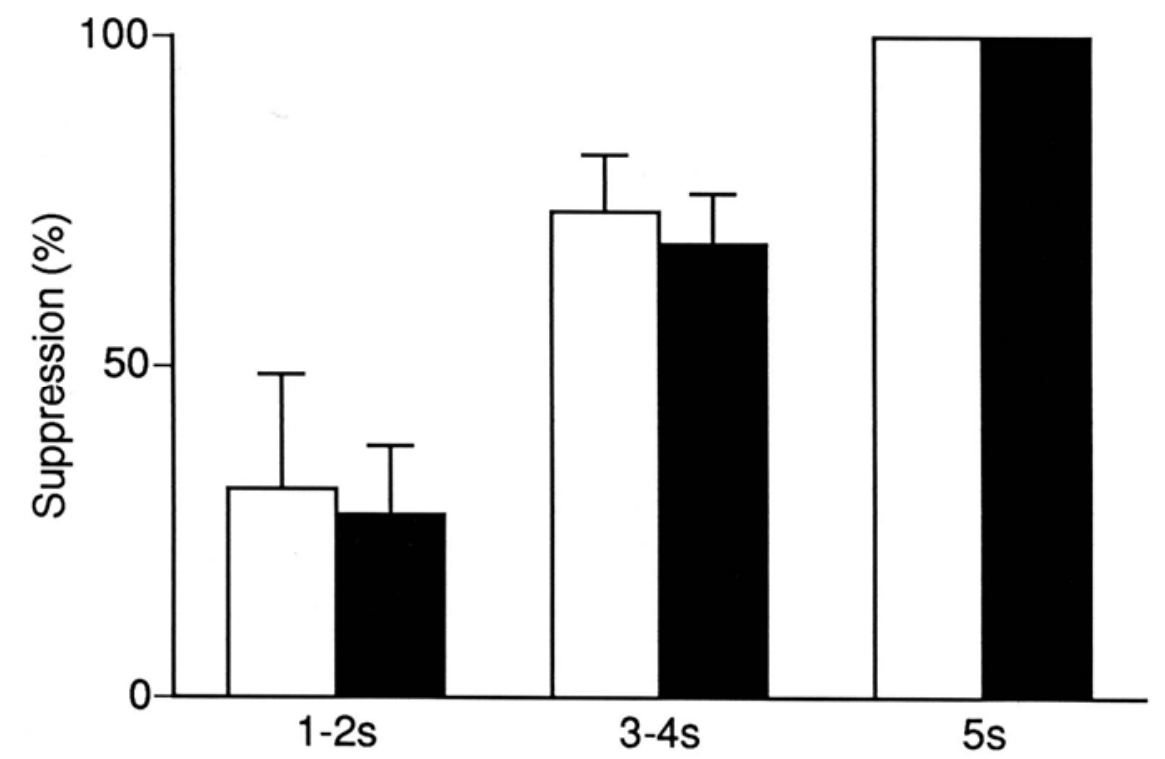

Figure 4 
A

control

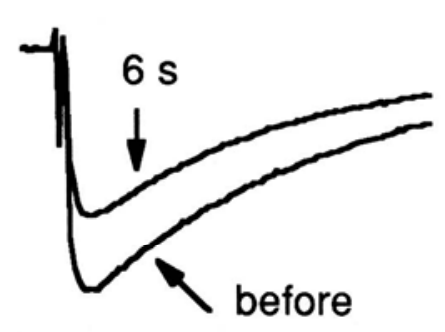

PDAC

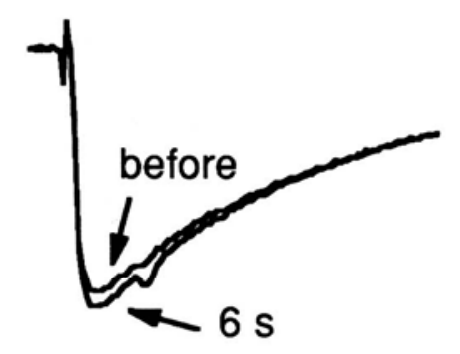

B

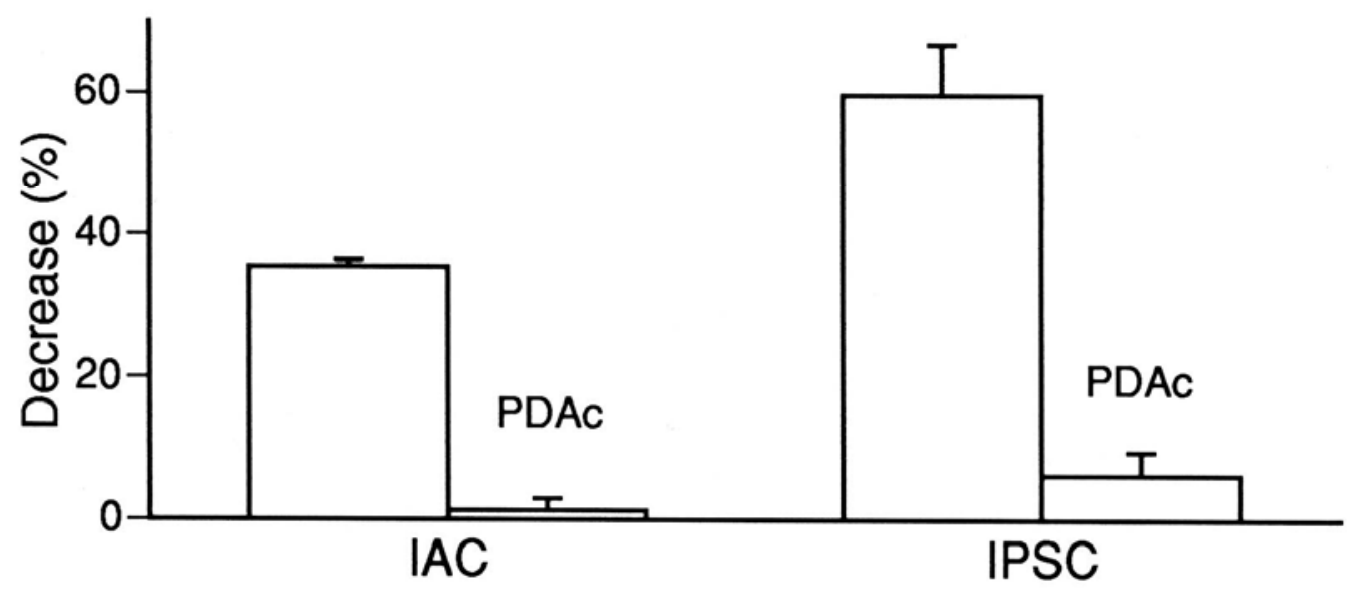

Figure 5

IPSC
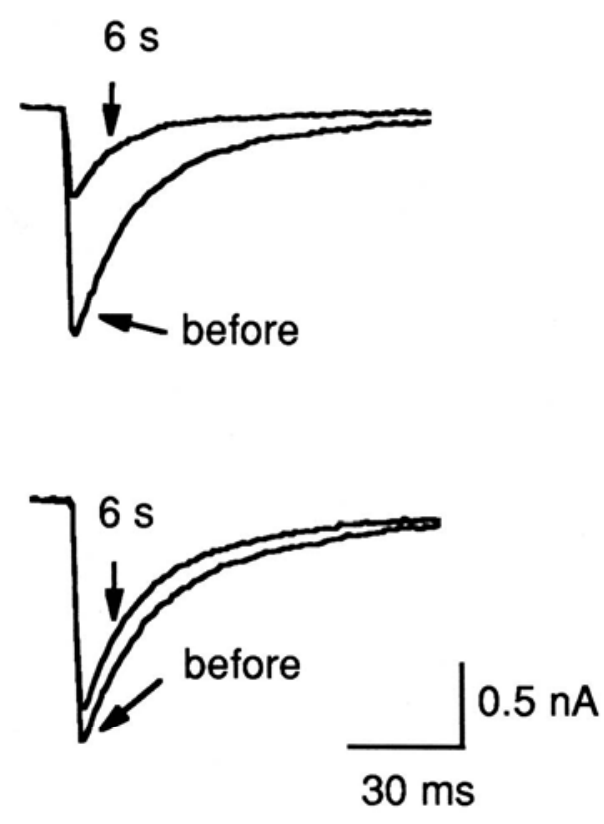
A

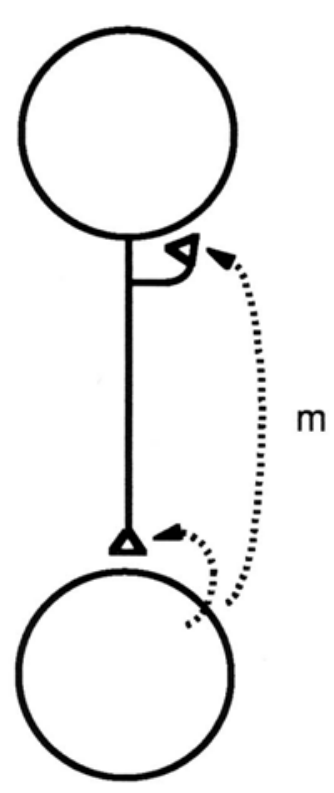

D

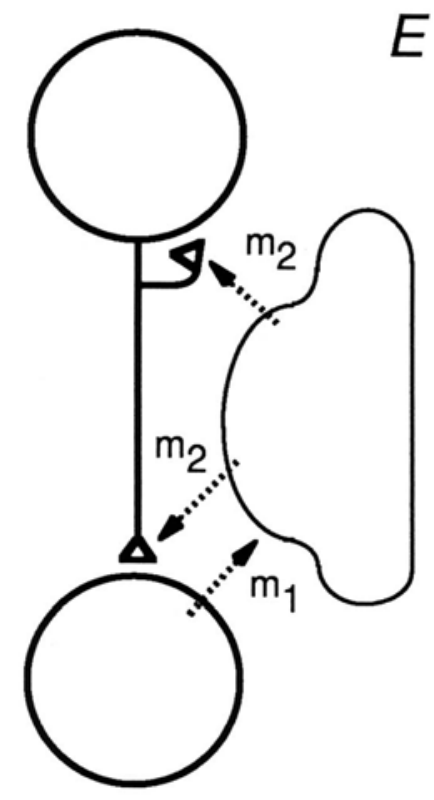

B

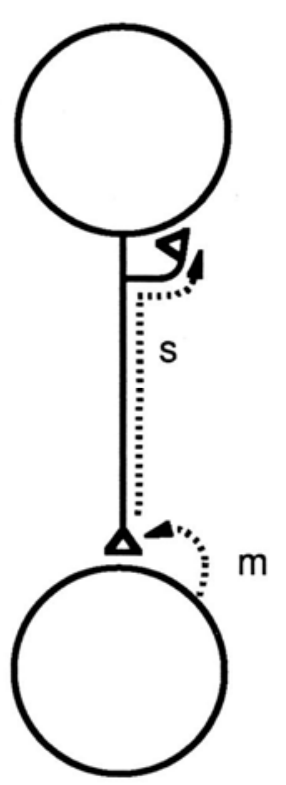

C

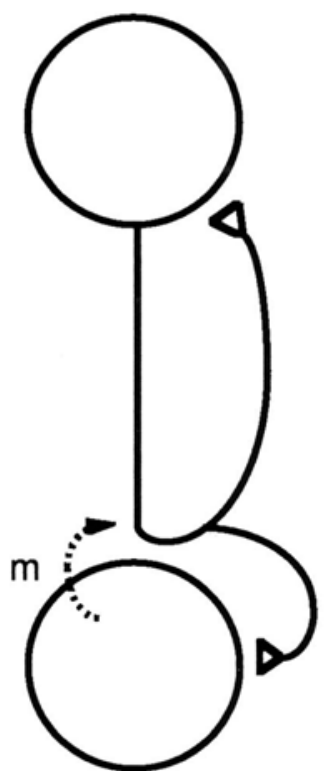

F
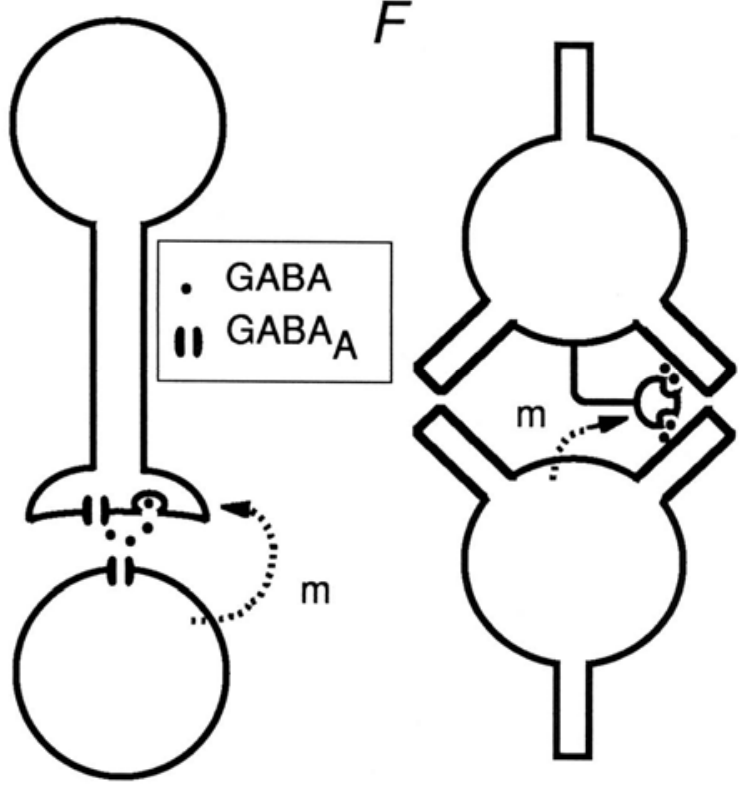

Figure 6 